\title{
Septic arthritis and Clostridium septicum: a clue to colon cancer
}

\author{
Joe Dylewski MD, Leonard Luterman MD
}

Previously published at www.cmaj.ca

A n 88-year-old woman reported acute-onset pain in her right knee after falling at home. While trying to get up from the toilet, she felt weak and fell, injuring her right knee and shoulder. A radiograph of her shoulder obtained the same day appeared normal, but a radiograph of her right knee showed prepatellar swelling of the soft tissue. Her knee was swollen and was warm to the touch. Cloudy yellow fluid was obtained by arthrocentesis, with a cell count of $71248 \times 10^{6} / \mathrm{L}$ neutrophils and $2496 \times 10^{6} / \mathrm{L}$ red blood cells. Gram staining showed over 100 white blood cells per low-power field, but no organisms were seen and the culture was negative.

The patient underwent arthroscopic debridement the following day, and grossly purulent material was sent for Gram staining and culture. The Gram stain showed many leukocytes but no organisms. However, after a two-day incubation period, anaerobic gram-positive rods, which formed a film on the media, were seen and identified as Clostridium septicum (Figure 1). The patient had originally been given intravenous cefazolin; this was subsequently changed to intravenous penicillin (three million units every four hours). A review of the laboratory data obtained on admission showed that she had normochromic normocytic anemia with a hemoglobin level of 103 (normal 120-160) g/L. Because of the results of the culture and her anemia, a colonoscopy was performed. A large ulcerated, invasive cecal adenocarcinoma was observed (Figure 2). Surgical resection of the adenocarcinoma was performed, and the patient completed a four-week course of intravenous penicillin. Repeat aspirations of the joint were negative for organisms at the end of treatment. The patient was discharged to a long-term care facility three months after admission. Adjuvant therapy for colonic cancer was not planned.

\section{Discussion}

Clostridium septicum is an aerotolerant gram-positive, gas-producing bacillus. It can be distinguished from the more common Clostridium perfringens by the presence of subterminal rather than central spores on Gram staining. Clostridium septicum is not considered part of the normal stool flora ${ }^{1,2}$ and is a rare cause of bacteremia, accounting for less than $1 \%$ of blood cultures that test positive for clostridia. ${ }^{3}$ The ability of the organism to invade and spread is aided by its flagella and the production of various enzymes, including fibrinolysin, hyaluronidase, deoxyribonuclease and two hemolysins. One of the hemolysins, $\alpha$-toxin

\section{Key points}

- Clostridium septicum is a gram-positive aerotolerant rod not usually present in the normal intestinal flora of humans. Infection with this organism is most commonly associated with spontaneous gas gangrene.

- Clostridium septicum infections are commonly associated with colonic or hematologic malignant growths.

- Treatment requires aggressive debridement of the infection site combined with intravenous antibiotics (penicillin, clindamycin or metronidazole).

- Clinicians should search for an underlying colonic cancer in cases of $C$. septicum infection.

(lecithinase), is responsible for the organism's virulence; it is 300 times more virulent than $C$. perfringens. ${ }^{4}$

\section{Pathogenesis}

Infection with $C$. septicum most commonly occurs after hematogenous dissemination, usually from ulceration of a colonic focus. ${ }^{5}$ However, there are also examples of soft-tissue infection after direct trauma. ${ }^{6}$ A recent review of the Englishlanguage literature dating back to 1969 found 320 cases of infection caused by $C$. septicum. ${ }^{7}$ In this review, $42 \%$ of cases had a gastrointestinal focus of the infection, with $39 \%$ being a malignant growth. Malignant disease of the colon is most commonly associated with this infection, which occurs in 30\%-50\% of cases. ${ }^{57,8}$ A possible explanation for this association is that the anaerobic glycolysis of the tumour may provide a hypoxic and acidic environment that favours germination of $C$. septicum spores. ${ }^{9}$ These areas of tissue necrosis may explain why hematologic and solid-organ malignant growths have also been reported in association with $C$. septicum. ${ }^{7}$ Concomitant bowel or hematologic malignant disease is associated with $80 \%-85 \%$ of all reported cases of $C$. septicum infection. ${ }^{5,7}$ Other anaerobic organisms have not been isolated more frequently under these circumstances; thus, the virulence factors must be important in the relation between $C$. septicum and the coexisting cancer. There is another clinical entity, Streptococcus gallolyticus (formerly bovis) bacteremia/endocarditis, that has been described in association with colonic cancer. ${ }^{10}$

From the Department of Medicine, St. Mary's Hospital Center, Montréal, Que.

CMAJ 2010. DOI:10.1503/cmaj.091946 


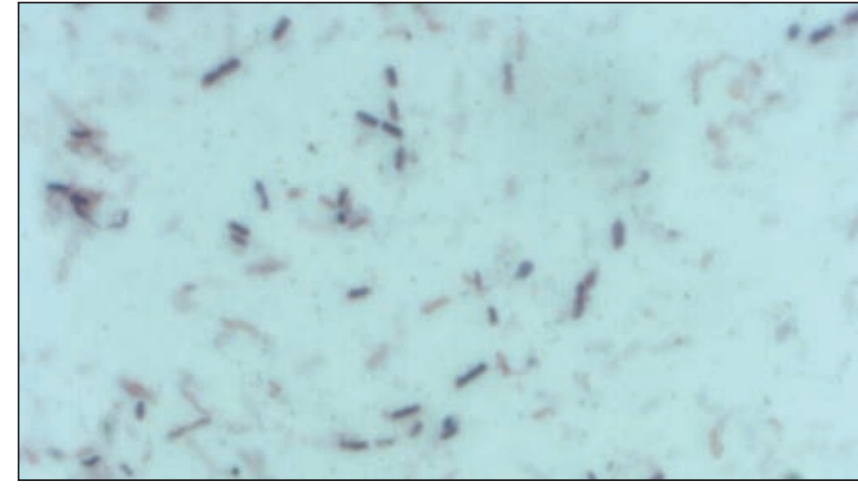

Figure 1: Gram stain of a colony of Clostridium septicum from a culture of knee fluid showing gram-positive rods (blue; original magnification $\times 1000$ ).

\section{Clinical presentation}

The most common clinical presentation of infection with C. septicum is skin and soft-tissue infection. ${ }^{6}$ One of the most serious manifestations is nontraumatic spontaneous gas gangrene, which has a high mortality rate. ${ }^{11}$ This diagnosis may not be evident early in the presentation because the involved skin may appear normal or only slightly edematous and tender. The infection progresses rapidly with bleb or bullae formation containing thin, nonpurulent fluid. The skin then becomes necrotic and changes to a purple-black colour. Gas can be detected by palpation or radiography. Management includes immediate surgery and high doses of antibiotics. ${ }^{4}$ The benefits of hyperbaric oxygen are unclear. ${ }^{12}$

Clostridium septicum is also associated with aortitis, ${ }^{13}$ which may be diagnosed by computed tomography. Aortitis is thought to occur by direct extension from a diseased colon or by hematogenous seeding of an atheromatous or aneurysmal aorta or both. In early aortitis, there is soft-tissue prominence with contrast enhancement around a normal-sized aorta. Air in and around the wall of the aorta is indicative of more advanced stage of infection. Treatment involves surgical resection of the infected section and graft replacement. A $75 \%$ six-month survival rate was reported in one series. ${ }^{13}$

Septic arthritis caused by anaerobic organisms is rare and accounts for $1 \%$ of cases of bacterial arthritis. In a 30-year review of published cases, Clostridium species were isolated in 37 cases of septic arthritis. ${ }^{8}$ We are aware of only five published cases of $C$. septicum-related arthritis; three of these patients also had colonic cancer. ${ }^{14-17}$ As in our patient, some patients did not have gas detected by radiography. ${ }^{6,15,16}$ Treatment consists of a high dose of penicillin given intravenously for at least four weeks and aggressive debridement of the affected joint. ${ }^{17}$ For patients who are allergic to penicillin, either clindamycin or metronidazole can be used. We recommend that clinicians search for an underlying colonic lesion, because the removal of gastrointestinal tumours is associated with higher survival rates.

This article has been peer reviewed.

Competing interests: None declared.

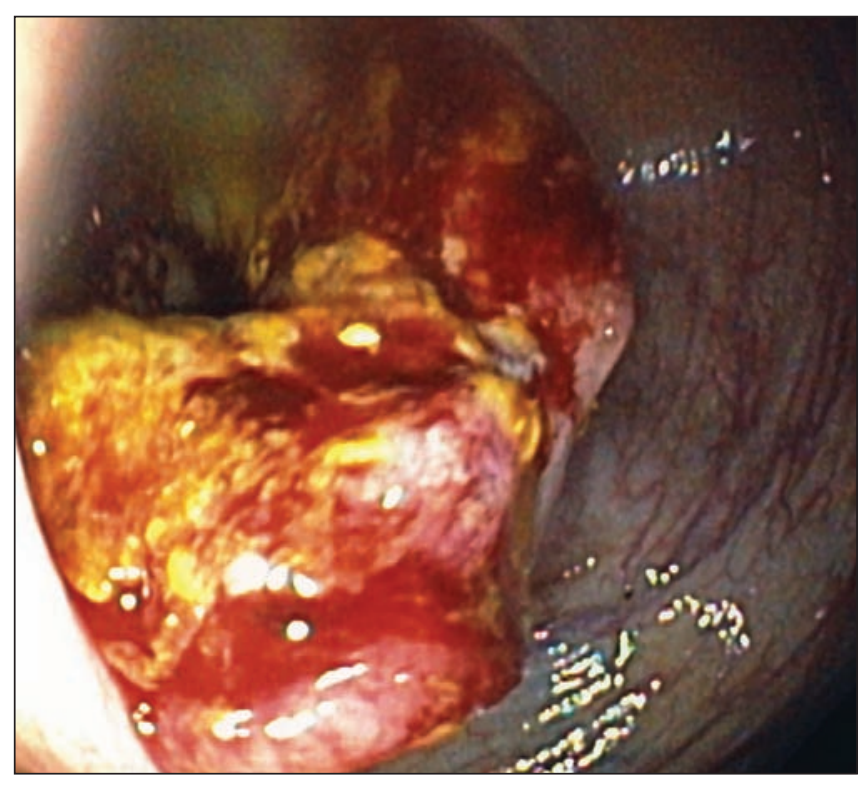

Figure 2: View from colonoscope showing a large ulcerated, tumour of the cecum with bleeding.

Contributors: Dr. Luterman performed the patient's colonoscopy and obtained the photograph of the colonic lesion. Drs. Dylewski and Luterman reviewed the literature. Dr. Dylewski wrote the manuscript, which was reviewed by Dr. Luterman. Both authors approved the final version of the manuscript submitted for publication.

\section{REFERENCES}

1. Moore WE, Holdeman LV. Human fecal flora: the normal flora of 20 JapaneseHawaiians. Appl Microbiol 1974;27:961-79.

2. Finegold SM, Attebery HR, Sutter VL. Effect of diet on human fecal flora: comparison of Japanese and American diets. Am J Clin Nutr 1974;27:1456-69.

3. Martin WJ. Isolation and identification of anaerobic bacteria in the clinical laboratory. A 2-year experience. Mayo Clin Proc 1974;49:300-8.

4. Stevens DL, Musher DM, Watson DA, et al. Spontaneous, nontraumatic gangrene due to Clostridium septicum. Rev Infect Dis 1990;12:286-96.

5. Kornbluth AA, Danzig JB, Berstein LH. Clostridium septicum infection and associated malignancy. Report of 2 cases and review of the literature. Medicine 1989;68:30-7.

6. Harris LF. Clostridium septicum arthritis. South Med J 1983;76:415.

7. Hermsen JL, Schurr MJ, Kudsk MD, et al. Phenotyping Clostridium septicum infection: a surgeon's infectious disease. J Surg Res 2008;148:67-76.

8. Gredlein CM, Silverman ML, Downey MS. Polymicrobial septic arthritis due to Clostridium species: case report and review. Clin Infect Dis 2000;30:590-4.

9. Alpern RJ, Dowell VR. Clostridium septicum infections and malignancy. JAMA 1969;209:385-8

10. Klein RS, Recco RA, Catalano MT, et al. Association of Streptococcus bovis with carcinoma of the colon. N Engl J Med 1977;297:800-2

11. Dylewski J, Drummond R, Rowen J. A case of Clostridium septicum spontaneous gas gangrene. CJEM 2007;9:133-5.

12. Sutherland ME, Meyer AA. Necrotizing soft-tissue infections. Surg Clin North Am 1994;74:591-607.

13. Seder CW, Kramer M, Long G, et al. Clostridium septicum aortitis: report of two cases and review of the literature. J Vasc Surg 2009;49:1304-9.

14. Fallon SM, Guzik HJ, Kramer LE. Clostridium septicum arthritis associated with colonic carcinoma. J Rheumatol 1986;13:662-3.

15. Macy NJ, Lieber L, Habermann ET. Arthritis caused by Clostridium septicum. A case report and review of the literature. J Bone Joint Surg Am 1986;68:465-6.

16. Hovenden JL, Murdoch GE, Evans AT. Non-traumatic Clostridium septicum arthritis in a patient with cecal carcinoma. Br J Rheumatol 1992;31:571-2.

17. Goon PKY, O'Brien M, Titley OG. Spontaneous Clostridium septicum septic arthritis of the shoulder and gas gangrene. J Bone Joint Surg Am 2005;87:874-7.

Correspondence to: Dr. Joe Dylewski, Department of Medicine,

St. Mary's Hospital Center, 3830 Lacombe, Montréal QC

H3T 1M5; joe.dylewski@ssss.gouv.qc.ca 